\title{
Survive or thrive: tradeoff strategy for cellular senescence
}

\author{
Sang Chul Park
}

Aging-dependent cellular behaviors toward extrinsic stress are characterized by the confined localization of certain molecules to either nuclear or perinuclear regions. Although most growth factors can activate downstream signaling in aging cells, they do not in fact have any impact on the cells because the signals cannot reach their genetic targets in the nucleus. For the same reason, varying apoptotic stress factors cannot stimulate the apoptotic pathway in senescent cells. Thus, the operation of a functional nuclear barrier in an aging-dependent manner has been investigated. To elucidate the mechanism for this process, the housekeeping transcription factor Sp1 was identified as a general regulator of nucleocytoplasmic trafficking (NCT) genes, including various nucleoporins, importins, exportins and Ran GTPase cycle-related genes. Interestingly, the posttranslational modification of Sp1 is readily influenced by extrinsic stress, including oxidative and metabolic stress. The decrease in SP1 O-GIcNAcylation under oxidative stress or during replicative senescence makes it susceptible to proteosomal degradation, resulting in defective NCT functions and leading to nuclear barrier formation. The operation of the nuclear barrier in aging provides a fundamental mechanism for cellular protection against stress and promotes survival at the expense of growth via stress-sensitive transcriptional control.

Experimental \& Molecular Medicine (2017) 49, e342; doi:10.1038/emm.2017.94; published online 2 June 2017

\section{INTRODUCTION}

Among the several characteristic features of senescent cells, including morphologic enlargement, senescence-associated $\beta$-galactosidase activity, reduced response to growth factors, increased apoptotic resistance and changes in gene expression, altered cellular responsiveness might be the most effective way to understand the phenotypic behaviors of senescent cells. ${ }^{1-4}$ Cellular responsiveness can be classified into three different categories. The first category is the metabolic response to nutrient supply and utilization. The metabolic response should be maintained intact for homeostasis regardless of age. The second category is the stress response against a variety of toxic stress. All cells and organisms are confronted with toxic or apoptotic stress. However, it is generally accepted that stress responses are attenuated with the age of the cell or organism. The third category is the mitogenic response. In aging, it is well known that responses to growth factors are distinctively reduced or even blocked. Responsiveness to stress and mitogenic factors is more attenuated or damaged than the metabolic response in aging cells and organisms. ${ }^{3}$ Therefore, it might be intriguing to elucidate the mechanism underlying these aging-dependent changes in cellular responsiveness.

\section{REDUCED RESPONSIVENESS TO GROWTH FACTORS: BARRIER TO THRIVE}

To determine cellular responsiveness, many aspects of its signaling system, such as ligand and receptor quantity, ligand-receptor affinity, downstream signaling cascades, intracellular networks, and signal translocation, transcriptional control, chromatin remodeling, and posttranscriptional status, should be assessed. ${ }^{4}$ Growth factors stimulate their respective receptors and subsequently activate downstream signals. However, senescent cells, in general, have a reduced efficiency of response to external growth factors. ${ }^{5-7}$ Senescent cells exhibit downregulation of mitogenic response toward EGF, despite questionable changes in the amounts of ligands or receptors. ${ }^{2,4,6,8}$ However, it has recently been shown that the functional recovery of senescent cells, especially with regard to the mitogenic response, could be induced to some extent simply through the adjustment of clathrin-dependent or -independent receptor-mediated endocytosis. ${ }^{9}$

The major component of caveolae is caveolin-1, which is abundant in terminally differentiated cell types and which is located at the sites where signaling molecules are concentrated. ${ }^{10-13}$ Caveolae, the subcellular compartments for

Well Aging Research Center, Department of New Biology, DGIST, Daegu, Korea

Correspondence: Professor SC Park, Department of New Biology, Well Aging Research Center, DGIST, 333 Joongangdae-ro, Hyeonpoong-myeon, Dalsung-gun, Daegu 42988, Korea.

E-mail: blueocean2016@dgist.ac.kr

Received 2 December 2016; revised 6 February 2017; accepted 16 February 2017 
storing and regulating signaling molecules, facilitate crosstalk between signaling cascades. The interaction of caveolin-1 with signaling molecules, such as $\mathrm{G}$ protein alpha-subunits, H-Ras, Src-family tyrosine kinases, PKC isoforms, EGFR, $\mathrm{Neu}$ and eNOS, is mediated via its membrane-proximal region, which is called the caveolin-scaffolding domain. ${ }^{14-17}$ The targeted downregulation of caveolin-1 is sufficient to drive the transformation of cells and to activate the Erk kinase cascade. Co-expression of EGFR with caveolin-1 results in the suppression of signal transduction from the cytoplasm to the nucleus in vivo. ${ }^{18}$ Senescent human diploid fibroblasts (HDFs) have an elevated level of caveolin-1, which colocalized with EGFR. ${ }^{5}$ Overexpression of caveolin-1 reduced the activation of Erk-1/2 after EGF stimulation, suggesting a direct role for caveolin-1 in EGF signaling, followed by the induction of premature cellular senescence of murine fibroblasts. ${ }^{4,19}$ By contrast, downregulation of caveolin-1 led to the restoration of basal p-Erk levels and Erk activation in response to EGF stimulation with the downstream activation of Elk phosphorylation in senescent HDFs. The activation of Erk signaling by its phosphorylation through PP1 and PP2A is also affected by caveolin- $1 .{ }^{20}$ Cell cycle arrest by caveolin-1 is controlled by the p53/p21Waf1-dependent pathway. $^{21}$ These results suggest the possibility of modulating the aging phenotype by the adjustment of the level of caveolin-1 in senescent cells. ${ }^{5}$

Furthermore, caveolin-1 is linked to the focal adhesion complex via integrins in the membrane, which implicates it in the control of focal adhesion and the adhesion signaling cascade. ${ }^{22-24}$ The formation of focal adhesion and actin stress fibers is relatively higher in senescent cells, and they are anchored to the membrane via interaction with caveolin-1. Therefore, the process of restoring the shape of senescent cells in order for them to resemble young cells by adjusting focal adhesion complexes via the control of caveolin-1 could be activated. $5,25,26$

The restoration of the senescent phenotype to a functionally active and young state by adjusting the caveolin-1 status illustrates the significant value of the membrane signaling system in aging. ${ }^{27}$ Therefore, it has been speculated that the aging process can be initiated and modulated at the membrane by a membrane-associated signal switch system. These data led to the emergence of the gate theory of aging, in which the fundamental role of a membrane switch system has been emphasized. 5,28 The gate theory of aging strongly suggests the possibility of restoration of the young phenotype in senescent cells by modulating the signaling system on the cell membrane. This novel hypothesis changes the conventional idea of aging as an irreversible and inevitable process into that of a flexible and plastic process. ${ }^{3,29}$

\section{ENHANCED RESISTANCE TO APOPTOTIC STRESS: STRATEGY TO SURVIVE}

Resistance to age-dependent apoptosis has been reported both in vitro and in vivo. ${ }^{30,31}$ Apoptosis in many physiological and pathological processes of aging and of age-related diseases is intimately connected to MAPKs and the serine/threonine kinases that phosphorylate specific substrates, respectively. ERK1 and ERK2 are well-characterized MAPKs that are activated mainly in response to growth stimuli, while JNKs and p38-MAPK are activated in response to a variety of stressors, including DNA damage, heat shock, ischemia, inflammatory cytokines, UV radiation and oxidative stress. ${ }^{32-37}$ The phosphorylation of MAPKs can be controlled in an agonist-specific manner. ${ }^{38-40}$ Hydrogen peroxide, an inducer of apoptosis, promotes an increase in ERK phosphorylation. ${ }^{41}$ Staurosporine, a strong inducer of caspase 3-dependent apoptosis, activates p38 phosphorylation. ${ }^{42}$ However, most of the signaling molecules can execute their respective functions only after their entry into the nucleus. Thus, it can be speculated that apoptotic resistance in senescent cells might be closely linked to defects of the apoptotic signals that prevent entry into nuclei. ${ }^{3}$

In mammalian cells, the ratio of proapoptotic proteins and anti-apoptotic Bcl-2 family members is important in determining whether and when apoptosis is triggered. ${ }^{43,44}$ The levels of $\mathrm{Bcl}-\mathrm{xL}$ and Bax were comparable in young and senescent HDFs, but Bak, Bok, Bik, and PUMA were present at lower levels in senescent HDFs than in young HDFs. ${ }^{3,40}$ These data imply that the lower level of expression of the proapoptotic genes might be related to the failure of the signaling molecules to trigger their expression, thereby desensitizing the senescent cells to apoptotic stimuli. By contrast, the stable expression of anti-apoptotic Bcl-2 in senescent cells despite apoptotic stress might be related to the phosphorylation status of CREB due to the inactivation of protein phophatase-2A ( $\mathrm{PP} 2 \mathrm{~A})$, as well as the inhibition of the apoptotic signal transduction into the nucleus. ${ }^{40}$ Moreover, the senescence-dependent nuclear accumulation of actin, gelsolin ${ }^{45,46}$ and major vault protein $(\mathrm{MVP})^{38}$ strongly support the specific localizing mechanism of the signal to either the cytosol or the nucleus. Therefore, we hypothesized that senescence-dependent defects in intracellular signaling, especially in nucleocytoplasmic trafficking, might provide a mechanism for cellular resistance to extrinsic stress. ${ }^{3}$

\section{DEFECTIVE NUCLEOCYTOPLASMIC TRAFFICKING: FROM FLEXIBILITY TO FIXATION}

Many signaling molecules and transcription factors need to enter the nucleus to exert their respective effects. For example, EGF stimulation induces the entry of p-ERK into the nucleus, which results in the activation of the transcription factor AP1, triggering cell cycle progression., 57 The apoptotic response is a well-programmed process that also requires the entry of intracellular signaling molecules into the nucleus. Therefore, it is natural to assume that the lack of responsiveness to both apoptotic stress and growth factors in senescence is related to the inefficiency of nucleocytoplasmic trafficking in the senescent cells. Nucleocytoplasmic trafficking is a highly sophisticated process that involves many component proteins. Nucleoporins are the major components of nuclear pore complexes (NPCs), often used as markers for NPCs. ${ }^{48,49}$ NPCs allow the passive diffusion of ions and small molecules, and 
facilitate the active transport of macromolecules. The cargo molecules usually have short sequence elements called nuclear localization sequences and nuclear export sequences. Karyopherin $\alpha$ binds to the nuclear localization sequences of cargo molecules, while karyopherin $\beta$ binds to both karyopherin $\alpha$ and nucleoporins..$^{50,51}$ Ran plays an important role in the import and export of cargoes, and is present in two distinct forms: GTP-bound Ran and GDP-bound Ran. The nucleotide state of Ran is regulated by Ran GTP-GDP exchange factor (RanGEF or RCC1) and Ran GTPase-activating protein (RanGAP). ${ }^{52,53}$

The initial observation of aging-dependent nuclear accumulation of actin $^{46}$ and gelsolin, ${ }^{45}$ as well as the perinuclear accumulation of many signaling molecules in response to a variety of apoptotic and mitotic stress conditions, ${ }^{39,40,54,55}$ implied that the nuclear translocation of activated signaling molecules might be inhibited by a certain barrier at the nuclear membrane, as shown by the perinuclear confinement of $\mathrm{p}$-ERK1/2 and the p50 subunit of NF-kB in response to growth stimuli or LPS in senescent fibroblasts. ${ }^{3,55}$ However, the activation of these signaling molecules is apparently not impaired, as illustrated by the efficient phosphorylation and activation of ERK1/2 via its interaction with PKC $\alpha$ or PP1 and PP2A in senescent cells. ${ }^{56-58}$ These findings assert that the signaling molecules cannot be distributed properly to the nucleus by simple activation but that they need the proper operation of the nucleocytoplasmic trafficking system under senescent conditions.

This nucleocytoplasmic trafficking system operates physiologically in response to external stimuli. In young and healthy cells, this system is readily influenced by either growth stimuli or nutritional condition. When the cellular energy state is low, the AMPK pathway is activated, resulting in the import of biomolecules into the nucleus. By contrast, when the cell receives growth stimuli, the PI $3 \mathrm{~K}$ signaling cascade is activated, leading to the export of biomolecules out of the nucleus, as demonstrated by GAPDH (glyceraldehyde 3-phosphate dehydrogenase). ${ }^{59}$ These data illustrate the plastic nature of nuclear translocation in cellular homeostasis. However, the operation of this trafficking system is strictly controlled in the senescent state. ${ }^{3}$

Many studies have been carried out on the structural and functional aspects of the nuclear trafficking system, as it is one of the essential features of metazoan life. ${ }^{60}$ Concerning the study of nuclear trafficking in aging, though the nature of its suppression and the reduction of NPC components have been acknowledged, the underlying mechanism for its loss of function remains unclear and disputed. ${ }^{61}$ The oxidative stress-dependent loss of nuclear trafficking by the oxidation of NPC components has previously been illustrated. ${ }^{62-64}$ Furthermore, aging-dependent leakage of the nuclear membrane has been suggested for the functional deterioration of nuclear trafficking. ${ }^{65,66}$ However, these reports are still unable to explain the aging-dependent dynamics of the nuclear trafficking system and the mechanistic regulation of functional hyporesponsiveness of senescent cells toward both growth and death-inducing stress.

\section{DYNAMICS OF NUCLEOCYTOPLASMIC TRAFFICKING: OPERATION OF THE NUCLEAR BARRIER}

Microarray analysis revealed that most of the nucleocytoplasmic trafficking genes, including most nucleoporin and transport receptor genes, as well as Ran and Ran-regulating factors, were downregulated in senescent HDFs. ${ }^{55}$ To verify the microarray data, the expression levels of some of the selected genes were confirmed with a semi-quantitative RT-PCR method and the protein levels were validated by western blotting with available antibodies. $^{54,55}$ These results showing the suppression of the nucleocytoplasmic trafficking genes and proteins in senescent HDFs strongly support the presumed defective operation of the nucleocytoplasmic trafficking system. ${ }^{3}$

The senescence-dependent reduction of nucleocytoplasmic trafficking gene expression was traced to its ultimate regulator through analysis of the upstream transcription factors for those genes. With bioinformatics tools, all the nucleocytoplasmic trafficking genes were subjected to promoter analysis for putative transcription factors. Among these transcription factors, Sp1 (specificity protein 1) was selected as the most common and dominant transcription factor responsible for the genetic control of the trafficking-associated genes. Furthermore, it was confirmed that most of the promoters of the trafficking-associated genes had multiple Sp1 binding sites. ${ }^{3,55}$

However, Spl stability was found to be damaged in senescent HDFs, and the DNA-binding activity of Sp1 was reduced in aged brain and liver tissues. ${ }^{67,68} \mathrm{Sp} 1$ protein levels were lower in senescent cells compared with young cells and in various tissues of aged mice compared with those from young mice. These results additionally support the possibility of Sp1 as a good candidate for the master regulation of NCT genes, showing that the reduced Sp1 protein levels in senescent cells and tissues could be a common causal factor for agingdependent suppression of the NCT genes. ${ }^{55,69}$

To test whether Sp1 was a common regulator for the expression of NCT genes, changes in NCT gene expression were determined after the modulation of Sp1 expression. Knockdown of Sp1 by si-Sp1 in young HDFs globally downregulated NCT genes. However, some nucleoporins, transport receptors and Ran GTPase cycle-related genes were heavily downregulated by Sp1 depletion, and the protein levels of Nup50, Nup88, Nup107, Nup155, karyopherin $\alpha 2$ and RCC1 were decreased in the Sp1-depleted HDFs. ${ }^{55}$ By contrast, Sp1 overexpression induced a general up-regulation of the NCT genes, and the protein levels of Nup50, Nup88, Nup107, Nup155, karyopherin $\alpha 2$ and RCC1 were also shown to be up-regulated. These results support the hypothesis that $\mathrm{Sp} 1$ is a common, functionally active master regulator of NCT gene expression. ${ }^{69}$ Additionally, the direct interaction of Sp1 with the promoter regions of NCT genes was confirmed by ChIP (chromatin immunoprecipitation) analysis (Ryu et al., submitted).

In order to determine the functional role of $\mathrm{Sp} 1$, the nuclear translocation of p-ERK1/2 was tested in response to EGF 


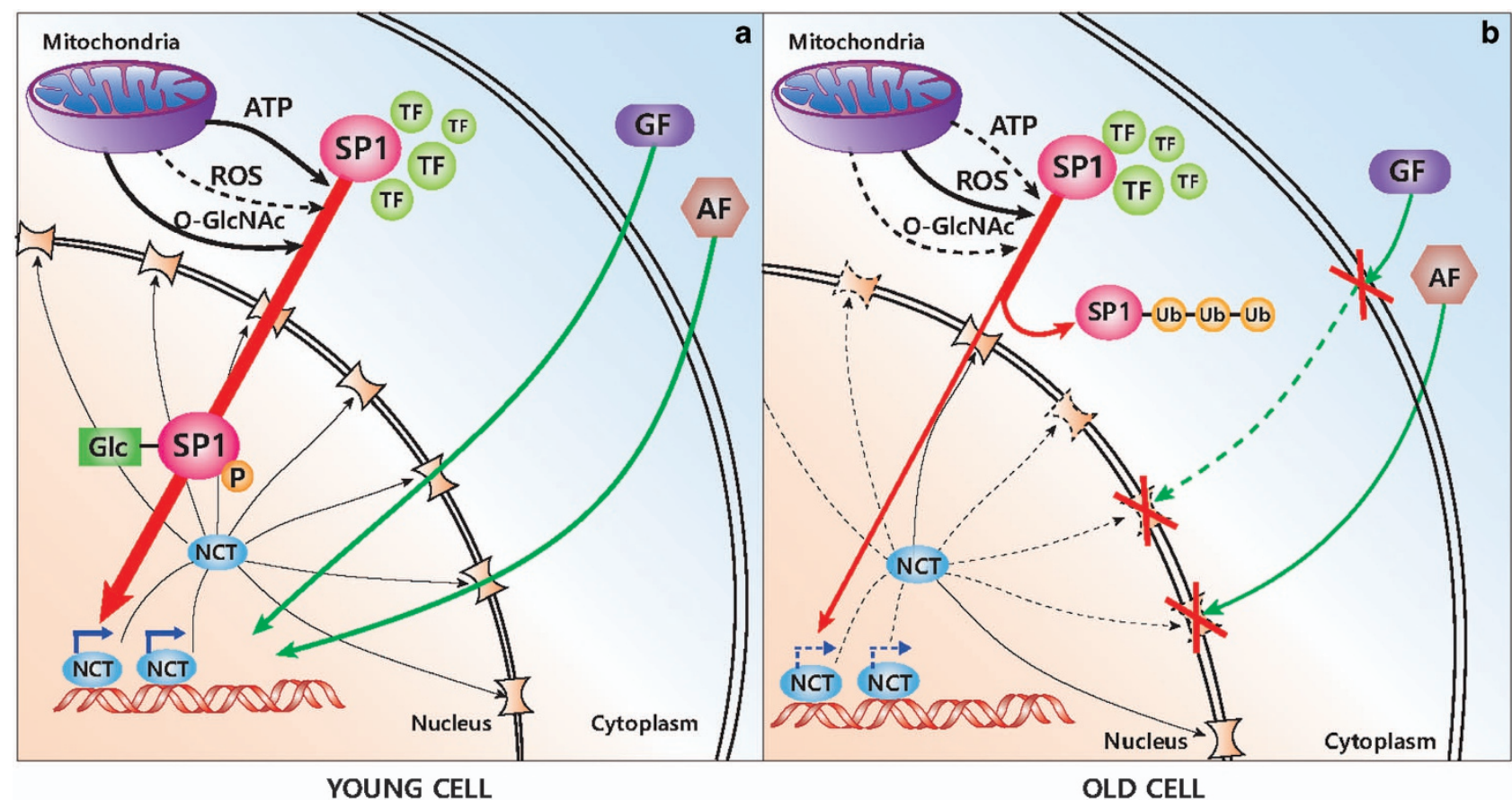

Figure 1 Comparison of nucleocytoplasmic trafficking between young and old cells. (a) Young cell. Sp1 can activate the transcription of NCT genes, and the nuclear pores are functioning well for both mitogenic and apoptotic signals. (b) Old cell. Sp1 is vulnerable to degradation, resulting in defective functioning of nuclear pores, which restricts signal trafficking. GF, growth factor; AF, apoptosis-inducing factor; TF, transcription factor; NCT, nucleocytoplasmic trafficking genes; O-GlcNAc, O-linked N-acetyl glucosamine; ub, ubiquitination; thick arrow, high activity; thin arrow, low activity; dotted line, restricted activity.

stimulation in either Sp1-depleted or Sp1-overexpressing cells. The majority of p-ERK1/2 molecules were present in the nuclear fraction after EGF stimulation in young $\mathrm{HDFs}$, while this nuclear localization was significantly attenuated in the si-Sp1-transfected cells. On the other hand, the majority of p-ERK1/2 was detected in the cytoplasmic fraction of senescent cells, and Sp1 overexpression significantly facilitated their nuclear localization. ${ }^{69}$ Furthermore, to confirm the role of Sp1 in the modulation of nuclear translocation of the signaling molecules, its downstream signaling was tested. Thus, the phosphorylation status of Elk, a known substrate of ERK1/2, and the expression of c-fos mRNA, a p-Elk target gene, were monitored. Elk phosphorylation at Serine 383 was markedly decreased in the Sp1-depleted cells compared with that in the young control cells, and was accompanied by a significant reduction of c-fos mRNA. These results strongly suggest that Sp1 influences the nucleocytoplasmic trafficking of signaling molecules, as well as their downstream signaling by modulating the efficiency of NCT gene expression. These data also strengthen the assumption that reduced Sp1 protein levels might be a causal factor for aging-dependent hyporesponsiveness to extrinsic stress. ${ }^{69}$

\section{MAINTENANCE OF THE NUCLEAR BARRIER IN AGING: REQUIREMENT FOR CONTINUING STRESS}

In order to identify the mechanism of downregulation of the Sp1 protein level under senescent conditions, the Sp1 mRNA level was determined, but no significant difference between young HDFs and senescent HDFs was obtained, implying the posttranslational regulation of Sp1 protein rather than its transcriptional control. As reactive oxygen species (ROS) levels are high in senescent HDFs, ${ }^{70,71} \mathrm{Sp} 1$ protein levels in response to $\mathrm{H}_{2} \mathrm{O}_{2}$ treatment were tested. The data demonstrated that the Sp1 protein level was strikingly reduced by $\mathrm{H}_{2} \mathrm{O}_{2}$ treatment, which was readily blocked by $\mathrm{N}$-acetylcysteine (NAC), a radical scavenger, supporting the hypothesis that the aging-related increase in ROS could be responsible for Sp1 protein downregulation. In addition, to determine whether the ROS-induced modification of the Sp1 protein was relevant for its proteasome-mediated degradation, Sp1 protein levels were determined with or without the proteasome inhibitor $\mathrm{N}-\mathrm{Ac}$-Leu-Leu-norleucinal (ALLN). The $\mathrm{H}_{2} \mathrm{O}_{2}$-dependent reduction of the Sp1 protein level was prevented by ALLN in both young and senescent HDFs, suggesting that Sp1 protein level is downregulated by ROS via proteasome-mediated degradation during the aging process. ${ }^{69}$

Because O-GlcNAcylation has been suggested as a mechanism for Sp1 stability, ${ }^{72}$ the $O$-GlcNAcylation status of Sp1 was compared between young and senescent HDFs. A marked decrease in the $O$-GlcNAcylation status of $\mathrm{Sp} 1$ was seen in senescent cells when compared with young cells. Furthermore, the level of O-GlcNAcylated Sp1 was significantly decreased by $\mathrm{H}_{2} \mathrm{O}_{2}$ treatment in the presence of ALLN. ${ }^{69}$ These data suggest that ROS can reduce the O-GlcNAcylation of Sp1, and can consequently facilitate its proteasome-mediated degradation in aging. In other words, the aging-related increase in ROS 
aggravates the hypo-O-GlcNAcylation of Sp1, followed by its consequent degradation. The dysfunction in glucose metabolism in senescent cells ${ }^{73}$ likely further limits the $\mathrm{O}$-GlcNAcylation of $\mathrm{Sp} 1$ to a low level in general by reducing hexosamine turnover, which requires high metabolic energy. ${ }^{69}$ This mechanism of aging-dependent nuclear barrier formation is summarized in Figure 1.

Sp1 is a transcription factor that has long been regarded as a regulator of housekeeping genes, and it has been well characterized for its structure and functions. Notably, its knockout in mice causes embryonic lethality with a broad range of phenotypic abnormalities, implicating a versatile function in many cell types. ${ }^{74-76}$ Chromosomal mapping studies of the human genome elucidated the presence of at least $12000 \mathrm{Sp} 1$ binding sites. ${ }^{77} \mathrm{Sp} 1$ is active in all cell types, and its activity is tightly regulated in response to signaling pathways and changing cellular conditions, which further affects its interaction with a variety of binding partners to regulate Sp1-dependent transcription. ${ }^{78,79}$ Considering the role of Sp1 in a multitude of cellular pathways and processes, it is natural to assume its association with the pathogenesis of a number of diseases, especially cancer. Sp1 overexpression has been observed in many cancer cell types, where the levels of Sp1 correlate with tumor stage, ${ }^{76}$ leading to the development of anticancer agents that inhibit the action of Sp1. ${ }^{80}$

However, the conflicting bifunctional behavior of Sp1 in cell growth and other biological phenomena limits our understanding of its genuine biological role. For example, Sp1 regulates the genes responsible for the progression of the cell cycle and entry into S-phase, such as cyclins and MYC, as well as growth factor signaling pathways, such as $\operatorname{IGF1R} .^{81,82}$ Sp1 also controls the transcription of cell cycle inhibitor genes, such as p21, synergizing with p53 under conditions of cellular stress. ${ }^{83,84}$ Moreover, the expression of telomerase subunits required for the maintenance of telomeres and cell immortality is also controlled by Sp1 through the Sp1 binding sites at the $h$ TERT promoter, ${ }^{85}$ while the interaction of SP1 with HDACs represses hTERT expression. ${ }^{86}$ Although only a few examples are illustrated here, $\mathrm{Spl}$ and its family members have a broad spectrum of biological functions operating in both pro- and anti-cell growth, which makes it difficult to discern their genuine biological functions. However, the novel concept of the role of Sp1 in the regulation of NCT function provides a new breakthrough in our understanding of its biological functions, as NCT is essential and strongly associated with most biological phenomena, including growth, differentiation, metabolism, apoptosis, cancer and senescence. Moreover, the dynamic nature of the $\mathrm{Spl}$ protein due to posttranslational modifications, such as phosphorylation, glycosylation, acetylation, poly (ADP-ribosyl)ation, methylation, sumoylation and oxidation, ${ }^{87-91}$ makes it sensitive to extrinsic stress, thereby implying its value as a regulator of biological responsiveness.

In addition, it is evident that many other transcription factors other than Sp1, as well as many NPC components, are also vulnerable to oxidative stress, leading to their loss of function. ${ }^{62-64}$ However, it can be assumed that the overall control of regulation of NPC formation by $\mathrm{Sp} 1$ is more powerful and effective for the aging process than the other individual components combined.

Taken together, it can be speculated that because Sp1 is one of the housekeeping genes, a continuous input of stress signals, whether metabolic or oxidative, would be required to maintain the reduced level of Sp1 protein, finally leading to senescence. All these data support the hypothesis that a functional nuclear barrier of hyporesponsiveness, called the 'Park and Lim's Barrier', to external stimuli is operating in senescent cells, in relation to the downregulation of the common transcription factor, Sp1. ${ }^{3}$ This Sp1 status-dependent nuclear barrier provides the mechanism of aging as a tradeoff between growth arrest and apoptosis resistance. The vulnerable nature of Sp1 protein status that is regulated by a variety of posttranslational modifications in response to nutritional supply and varying stress further supports the role of Spl as one of the prime determinants of the senescent phenotype. In addition, it can be speculated that, through the adjustment of Sp1 status, the nucleocytoplasmic trafficking defects of senescent cells can be restored and the aging phenotype can be reversed. It has already been shown that Spl overexpression can induce the restoration of the telomere damage-dependent senescent phenotype to a young phenotype to some extent. ${ }^{92}$ Therefore, there is no doubt that the modulation of this barrier mechanism simply via changing Sp1 status may open a new avenue for the adjustment of nuclear trafficking in senescence, cancers and neurodegenerative disorders.

\section{CONCLUSION}

The features of hypo-responsiveness of senescent cells either to growth factors or to apoptotic stress are related to a functional nuclear barrier with defective nucleocytoplasmic trafficking resulting from the transcriptional downregulation of the relevant genes. This barrier is important for the survival of the aging cells at the expense of growth and provides a platform for regulating the crosstalk between good and bad signals from extrinsic sources. In addition, the transcription factor Sp1 has been implicated in the regulation of this barrier, and, through simple adjustments of Sp1 levels, the nucleocytoplasmic trafficking defects of senescent cells can be restored, and consequently, the aging phenotype can be reversed. Furthermore, it can be assumed that this mechanism may provide new insights for novel therapeutic modalities in cancers, neurodegenerative disorders and other aging-related diseases by adjusting the nucleocytoplasmic trafficking status.

\section{CONFLICT OF INTEREST}

The author declares no conflict of interest.

\section{ACKNOWLEDGEMENTS}

This manuscript is sincerely dedicated to Drs Earl \& Terry Stadtman and Dr Sue Goo Rhee for their great contribution to encourage Korean young biochemists. This research was supported by the DGIST 
R\&D Program of the Ministry of Science, ICT and Technology of KOREA (20160172).

1 Dimri GP, Lee XH, Basile G, Acosta M, Scott C, Roskelley C et al. A biomarker that identifies senescent human-cells in culture and in aging skin in-vivo. Proc Natl Acad Sci USA 1995; 92: 9363-9367.

2 Park SC. Functional recovery of senescent cells through restoration of receptor-mediated endocytosis. Mech Ageing Dev 2002; 123: 917-926.

3 Park SC. Nuclear barrier hypothesis of aging as mechanism for trade-off growth to survival. Adv Exp Med Biol 2011; 720: 3-13.

4 Park WY, Park JS, Cho KA, Kim DI, Ko YG, Seo JS et al. Up-regulation of caveolin attenuates epidermal growth factor signaling in senescent cells. J Biol Chem 2000; 275: 20847-20852.

5 Cho KA, Park SC. Caveolin-1 as a prime modulator of aging: a new modality for phenotypic restoration? Mech Ageing Dev 2005; 126: 105-110.

6 Phillips PD, Kuhnle E, Cristofalo VJ. [I-125]-Labeled Egf Binding Ability Is Stable Throughout the Replicative Life-Span of Wi-38 Cells. J Cell Physiol 1983; 114: 311-316.

7 Yeo EJ, Jang IS, Lim HK, Ha KS, Park SC. Agonist-specific differential changes of cellular signal transduction pathways in senescent human diploid fibroblasts. Exp Gerontol 2002; 37: 871-883.

8 Carlin CR, Phillips PD, Knowles BB, Cristofalo VJ. Diminished in vitro tyrosine kinase activity of the EGF receptor of senescent human fibroblasts. Nature 1983; 306: 617-620.

9 Park JS, Park WY, Cho KA, Kim DI, Jhun BH, Kim SR et al. Down-regulation of amphiphysin-1 is responsible for reduced receptormediated endocytosis in the senescent cells. FASEB J 2001; 15: 1625-1627.

10 Anderson RG. The caveolae membrane system. Annu Rev Biochem 1998; 67: 199-225.

11 Bretscher MS, Whytock S. Membrane-Associated Vesicles in Fibroblasts. J Ultra Mol Struct R 1977; 61: 215-217.

12 Okamoto T, Schlegel A, Scherer PE, Lisanti MP. Caveolins, a family of scaffolding proteins for organizing 'preassembled signaling complexes' at the plasma membrane'. J Biol Chem 1998; 273: 5419-5422.

13 Sargiacomo M, Scherer PE, Tang Z, Kubler E, Song KS, Sanders MC et al. Oligomeric structure of caveolin: implications for caveolae membrane organization. Proc Natl Acad Sci USA 1995; 92: 9407-9411.

14 Couet J, Li SW, Okamoto T, Ikezu T, Lisanti MP. Identification of peptide and protein ligands for the caveolin-scaffolding domain-implications for the interaction of caveolin with caveolae-associated proteins. J Biol Chem 1997; 272: 6525-6533.

15 Couet J, Sargiacomo M, Lisanti MP. Interaction of a receptor tyrosine kinase, EGF-R, with caveolins-Caveolin binding negatively regulates tyrosine and serine/threonine kinase activities. J Biol Chem 1997; 272: 30429-30438.

16 Li SW, Couet J, Lisanti MP. Src tyrosine kinases, G(alpha) subunits, and $\mathrm{H}$-Ras share a common membrane-anchored scaffolding protein, caveolin - Caveolin binding negatively regulates the auto-activation of Src tyrosine kinases. J Biol Chem 1996; 271: 29182-29190.

17 Song KS, Li S, Okamoto T, Quilliam LA, Sargiacomo M, Lisanti MP. Co-purification and direct interaction of Ras with caveolin, an integral membrane protein of caveolae microdomains. Detergent-free purification of caveolae microdomains. J Biol Chem 1996; 271: 9690-9697.

18 Engelman JA, Chu $\mathrm{C}$, Lin $\mathrm{A}$, Jo $\mathrm{H}$, Ikezu $\mathrm{T}$, Okamoto $\mathrm{T}$ et al. Caveolin-mediated regulation of signaling along the p42/44 MAP kinase cascade in vivo - A role for the caveolin-scaffolding domain. Febs Lett 1998; 428: 205-211.

19 Volonte D, Zhang K, Lisanti MP, Galbiati F. Expression of caveolin-1 induces premature cellular senescence in primary cultures of murine fibroblasts-Stress-induced premature senescence upregulates the expression of endogenous caveolin-1. Mol Biol Cell 2002; 13: 2502-2517.

20 Li LK, Ren CH, Tahir SA, Ren CZ, Thompson TC. Caveolin-1 maintains activated Akt in prostate cancer cells through scaffolding domain binding site interactions with and inhibition of serine/threonine protein phosphatases PP1 and PP2A. Mol Cell Biol 2003; 23: 9389-9404.

21 Galbiati F, Volonte D, Liu J, Capozza F, Frank PG, Zhu L et al. Caveolin-1 expression negatively regulates cell cycle progression by inducing $\mathrm{G}(0) / \mathrm{G}(1)$ arrest via a p53/p21(WAF1/Cip1)-dependent mechanism. Mol Biol Cell 2001; 12: 2229-2244.
22 Chapman HA, Wei Y, Simon DI, Waltz DA. Role of urokinase receptor and caveolin in regulation of integrin signaling. Thromb Haemost 1999; 82: 291-297.

23 Teixeira A, Chaverot N, Schroder C, Strosberg AD, Couraud PO, Cazaubon $\mathrm{S}$. Requirement of caveolae microdomains in extracellular signal-regulated kinase and focal adhesion kinase activation induced by endothelin-1 in primary astrocytes. J Neurochem 1999; 72: 120-128.

24 Wei Y, Yang XW, Liu QM, Wilkins JA, Chapman HA. A role for caveolin and the urokinase receptor in integrin-mediated adhesion and signaling. J Cell Biol 1999; 144: 1285-1294.

25 Cho KA, Ryu SJ, Oh YS, Park JH, Lee JW, Kim HP et al. Morphological adjustment of senescent cells by modulating caveolin- 1 status. J Biol Chem 2004; 279: 42270-42278.

26 Cho KA, Ryu SJ, Park JS, Jang IS, Ahn JS, Kim KT et al. Senescent phenotype can be reversed by reduction of caveolin status. J Biol Chem 2003; 278: 27789-27795.

27 Park SC, Cho KA, Jang IS, Kim KT, Ryu SJ. Functional efficiency of the senescent cells: Replace or restore? Ann Ny Acad Sci 2004; 1019: 309-316.

28 Yeo EJ, Park SC. Age-dependent agonist-specific dysregulation of membrane-mediated signal transduction: emergence of the gate theory of aging. Mech Ageing Dev 2002; 123: 1563-1578.

29 Park SC. New molecular target for modulation of aging process. Antioxid Redox Sign 2006; 8: 620-627.

30 Suh Y, Lee KA, Kim WH, Han BG, Vijg J, Park SC. Aging alters the apoptotic response to genotoxic stress. Nat Med 2002; 8: 3-4.

31 Yeo EJ, Hwang YC, Kang CM, Choy HE, Park SC. Reduction of UV-induced cell death in the human senescent fibroblasts. Mol Cells 2000; 10: 415-422.

32 Chang L, Karin M. Mammalian MAP kinase signalling cascades. Nature 2001; 410: 37-40.

33 Dent $\mathrm{P}$, Yacoub A, Fisher PB, Hagan MP, Grant S. MAPK pathways in radiation responses. Oncogene 2003; 22: 5885-5896.

34 Johnson GL, Lapadat R. Mitogen-activated protein kinase pathways mediated by ERK, JNK, and p38 protein kinases. Science 2002; 298: 1911-1912.

35 Royuela M, Arenas MI, Bethencourt FR, Sanchez-Chapado M, Fraile B, Paniagua R. Regulation of proliferation/apoptosis equilibrium by mitogenactivated protein kinases in normal, hyperplastic, and carcinomatous human prostate. Hum Pathol 2002; 33: 299-306.

36 Seger R, Krebs EG. The MAPK signaling cascade. FASEB J 1995; 9: 726-735.

37 Seluanov A, Gorbunova V, Falcovitz A, Sigal A, Milyavsky M, Zurer I et al. Change of the death pathway in senescent human fibroblasts in response to DNA damage is caused by an inability to stabilize p53. Mol Cell Biol 2001; 21: 1552-1564.

38 Ryu SJ, An HJ, Oh YS, Choi HR, Ha MK, Park SC. On the role of major vault protein in the resistance of senescent human diploid fibroblasts to apoptosis. Cell Death Differ 2008; 15: 1673-1680.

39 Ryu SJ, Cho KA, Oh YS, Park SC. Role of Src-specific phosphorylation site on focal adhesion kinase for senescence-associated apoptosis resistance. Apoptosis 2006; 11: 303-313.

40 Ryu SJ, Oh YS, Park SC. Failure of stress-induced downregulation of Bcl-2 contributes to apoptosis resistance in senescent human diploid fibroblasts. Cell Death Differ 2007; 14: 1020-1028.

41 Martin D, Salinas M, Fujita N, Tsuruo T, Cuadrado A. Ceramide and reactive oxygen species generated by $\mathrm{H} 2 \mathrm{O} 2$ induce caspase-3-independent degradation of Akt/protein kinase B. J Biol Chem 2002; 277: 42943-42952.

42 Tesauro M, Thompson WC, Moss J. Effect of staurosporine-induced apoptosis on endothelial nitric oxide synthase in transfected COS-7 cells and primary endothelial cells. Cell Death Differ 2006; 13: 597-606.

43 Kuwana T, Newmeyer DD. Bcl-2-family proteins and the role of mitochondria in apoptosis. Curr Opin Cell Biol 2003; 15: 691-699.

44 Pepper C, Bentley P. The role of the Bcl-2 family in the modulation of apoptosis. Symp Soc Exp Biol 2000; 52: 43-53.

45 Ahn JS, Jang IS, Kim DI, Cho KA, Park YH, Kim K et al. Aging-associated increase of gelsolin for apoptosis resistance. Biochem Biophys Res Commun 2003; 312: 1335-1341.

46 Lim IK, Hong KW, Kwak IH, Yoon G, Park SC. Cytoplasmic retention of $\mathrm{p}$-Erk1/2 and nuclear accumulation of actin proteins during cellular senescence in human diploid fibroblasts. Mech Ageing Dev 2000; 119: 113-129.

47 Kim-Kaneyama JR, Nose K, Shibanuma M. Significance of nuclear relocalization of ERK1/2 in reactivation of $\mathrm{c}$-fos transcription and DNA 
synthesis in senescent fibroblasts. J Biol Chem 2000; 275: 20685-20692.

48 Beck M, Forster F, Ecke M, Plitzko JM, Melchior F, Gerisch G et al. Nuclear pore complex structure and dynamics revealed by cryoelectron tomography. Science 2004; 306: 1387-1390.

49 Stoffler D, Fahrenkrog B, Aebi U. The nuclear pore complex: from molecular architecture to functional dynamics. Curr Opin Cell Biol 1999; 11: 391-401.

50 D'Angelo MA, Hetzer MW. Structure, dynamics and function of nuclear pore complexes. Trends Cell Biol 2008; 18: 456-466.

51 Hoelz A, Blobel G. Cell biology_Popping out of the nucleus. Nature 2004; 432: 815-816.

52 Izaurralde E, Kutay U, vonKobbe C, Mattaj IW, Gorlich D. The asymmetric distribution of the constituents of the Ran system is essential for transport into and out of the nucleus. EMBO J 1997; 16: 6535-6547.

53 Turpin P, Ossareh-Nazari B, Dargemont C. Nuclear transport and transcriptional regulation. Febs Lett 1999; 452: 82-86.

54 Kim SY, Kang HT, Choi HR, Park SC. Reduction of Nup107 attenuates the growth factor signaling in the senescent cells. Biochem Bioph Res Co 2010; 401: 131-136.

55 Kim SY, Ryu SJ, Ahn HJ, Choi HR, Kang HT, Park SC. Senescence-related functional nuclear barrier by down-regulation of nucleo-cytoplasmic trafficking gene expression. Biochem Bioph Res Co 2010; 391: 28-32.

56 Kim HS, Song MC, Kwak IH, Park TJ, Lim IK. Constitutive induction of p-Erk $1 / 2$ accompanied by reduced activities of protein phosphatases 1 and $2 \mathrm{~A}$ and MKP3 due to reactive oxygen species during cellular senescence. J Biol Chem 2003; 278: 37497-37510.

57 Kim HS, Lim IK. Phosphorylated extracellular signal-regulated protein kinases 1 and 2 phosphorylate Sp1 on serine 59 and regulate cellular senescence via transcription of p21Sdi1/Cip1/Waf1. J Biol Chem 2009; 284: 15475-15486.

58 Lee YY, Ryu MS, Kim HS, Suganuma M, Song KY, Lim IK. Regulations of Reversal of Senescence by PKC Isozymes in Response to 12-0-Tetradecanoylphorbol-13-Acetate via Nuclear Translocation of pErk1/2. Mol Cells 2016; 39: 266-279.

59 Kwon HJ, Rhim JH, Jang IS, Kim GE, Park SC, Yeo EJ. Activation of AMP-activated protein kinase stimulates the nuclear localization of glyceraldehyde 3-phosphate dehydrogenase in human diploid fibroblasts. Exp Mol Med 2010; 42: 254-269.

60 Kodiha M, Crampton N, Shrivastava S, Umar R, Stochaj U. Traffic control at the nuclear pore. Nucleus-Austin 2010; 1: 237-244.

61 Fichtman B, Harel A. Stress and aging at the nuclear gateway. Mech Ageing Dev 2014; 135: 24-32.

62 Chatterjee M, Paschal BM. Disruption of the ran system by cysteine oxidation of the nucleotide exchange factor RCC1. Mol Cell Biol 2015; 35: 566-581.

63 Datta S, Snow CJ, Paschal BM. A pathway linking oxidative stress and the Ran GTPase system in progeria. Mol Biol Cell 2014; 25: 1202-1215.

64 Kodiha M, Stochaj U. Nuclear transport: a switch for the oxidative stresssignaling circuit? J Signal Transduct 2012; 2012: 208650.

65 Hatch E, Hetzer M. Breaching the nuclear, envelope in development and disease. J Cell Biol 2014; 205: 133-141.

66 Hatch EM, Hetzer MW. Nuclear envelope rupture is induced by actin-based nucleus confinement. J Cell Biol 2016; 215: 27-36.

67 Kang HT, Ju JW, Cho JW, Hwang ES. Down-regulation of Sp1 activity through modulation of O-glycosylation by treatment with a low glucose mimetic, 2-deoxyglucose. J Biol Chem 2003; 278: 51223-51231.

$68 \mathrm{Oh} \mathrm{JE}$, Han JA, Hwang ES. Downregulation of transcription factor, Sp1, during cellular senescence. Biochem Bioph Res Co 2007; 353: 86-91.

$69 \mathrm{Kim}$ SY, Kang HT, Han JA, Park SC. The transcription factor Spl is responsible for aging-dependent altered nucleocytoplasmic trafficking. Aging Cell 2012; 11: 1102-1109.

70 Beckman KB, Ames BN. The free radical theory of aging matures. Physiol Rev 1998; 78: 547-581.

71 Sohal RS, Weindruch R. Oxidative stress, caloric restriction, and aging. Science 1996; 273: 59-63.

72 Han I, Kudlow JE. Reduced 0 glycosylation of Sp1 is associated with increased proteasome susceptibility. Mol Cell Biol 1997; 17: 2550-2558.

73 Zwerschke W, Mazurek S, StockI P, Hutter E, Eigenbrodt E, Jansen-Durr P. Metabolic analysis of senescent human fibroblasts reveals a role for AMP in cellular senescence. Biochem J 2003; 376: 403-411.
74 Briggs MR, Kadonaga JT, Bell SP, Tjian R. Purification and biochemical characterization of the promoter-specific transcription factor, Sp1. Science 1986; 234: 47-52.

75 Marin M, Karis A, visser P, Grosveld F, Philipsen S. Transcription factor $\mathrm{Sp} 1$ is essential for early embryonic development but dispensable for cell growth and differentiation. Cell 1997; 89: 619-628.

$76 \mathrm{Li} \mathrm{L,} \mathrm{Davie} \mathrm{JR.} \mathrm{The} \mathrm{role} \mathrm{of} \mathrm{Sp1} \mathrm{and} \mathrm{Sp3} \mathrm{in} \mathrm{normal} \mathrm{and} \mathrm{cancer} \mathrm{cell} \mathrm{biology.}$ Ann Anat 2010; 192: 275-283.

77 Cawley S, Bekiranov S, Ng HH, Kapranov P, Sekinger EA, Kampa D et al. Unbiased mapping of transcription factor binding sites along human chromosomes 21 and 22 points to widespread regulation of noncoding RNAs. Cell 2004; 116: 499-509.

78 Wierstra I. Sp1: emerging roles-Beyond constitutive activation of TATA-less housekeeping genes. Biochem Biophys Res Commun 2008; 372: 1-13.

79 Beishline K, Azizkhan-Clifford J. Sp1 and the 'hallmarks of cancer'. FEBS J 2015; 282: 224-258.

80 Vizcaino C, Mansilla S, Portugal J. Sp1 transcription factor: a long-standing target in cancer chemotherapy. Pharmacol Ther 2015; 152: 111-124.

81 Chitnis MM, Yuen JS, Protheroe AS, Pollak M, Macaulay VM. The type 1 insulin-like growth factor receptor pathway. Clin Cancer Res 2008; 14: 6364-6370.

82 Zhu JL, Kaytor EN, Pao Cl, Meng XP, Phillips LS. Involvement of Sp1 in the transcriptional regulation of the rat insulin-like growth factor-1 gene. Mol Cell Endocrinol 2000; 164: 205-218.

83 Kyo S, Takakura M, Taira T, Kanaya T, Itoh H, Yutsudo M et al. Sp1 cooperates with c-Myc to activate transcription of the human telomerase reverse transcriptase gene (hTeRT). Nucleic Acids Res 2000; 28: 669-677.

84 Lagger G, Doetzlhofer A, Schuettengruber B, Haidweger E, Simboeck E, Tischler $\mathrm{J}$ et al. The tumor suppressor p53 and histone deacetylase 1 are antagonistic regulators of the cyclin-dependent kinase inhibitor p21/WAF1/ CIP1 Gene. Mol Cell Biol 2003; 23: 2669-2679.

85 Huang L, Sowa Y, Sakai T, Pardee AB. Activation of the p21WAF1/CIP1 promoter independent of $\mathrm{p} 53$ by the histone deacetylase inhibitor suberoylanilide hydroxamic acid(SAHA) through the Sp1 sites. Oncogene 2000; 19: 5712-5719.

86 Won J, Yim J, Kim TK. Sp1 and Sp3 recruit histone deacetylase to repress transcription of human telomerase reverse transcriptase (hTeRT) promoter in normal human. somatic cells. J Biol Chem 2002; 277: 38230-38238.

87 Yang X, Su K, Roos MD, Chang Q, Paterson AJ, Kudlow JE. O-linkage of $\mathrm{N}$-acetylglucosamine to $\mathrm{Sp} 1$ activation domain inhibits its transcriptional capability. Proc Natl Acad Sci USA 2001; 98: 6611-6616.

88 Zaniolo K, Desnoyers S, Leclerc S, Guerin SL. Regulation of poly(ADP-ribose) polymerase-1 (PARP-1) gene expression through the post-translational modification of Sp1: a nuclear target protein of PARP-1. BMC Mol Biol 2007; 8: 96-113.

89 Chuang JY, Chang WC, Hung JJ. Hydrogen peroxide induces Sp1 methylation and thereby suppresses cyclin B1 via recruitment of Suv39H1 and HDAC1 in cancer cells. Free Radic Biol Med 2011; 51: 2309-2318.

90 Spengler ML, Brattain MG. Sumoylation inhibits cleavage of Sp1 $\mathrm{N}$-terminal negative regulatory domain and inhibits Sp1-dependent transcription. J Biol Chem 2006; 281: 5567-5574.

$91 \mathrm{Chu}$ S. Transcriptional regulation by post-transcriptional modification-role of phosphorylation in Sp1 transcriptional activity. Gene 2012; 508: 1-8.

92 An HJ, Lee HJ, Jang S, Jung YJ, Choi SS, Park SC et al. Transcription factor Sp1 prevents TRF2(Delta B Delta M)-induced premature senescence in human diploid fibroblasts. Mol Cell Biochem 2016; 414: 201-208.

(1) This work is licensed under a Creative Commons Attribution 4.0 International License. The images or other third party material in this article are included in the article's Creative Commons license, unless indicated otherwise in the credit line; if the material is not included under the Creative Commons license, users will need to obtain permission from the license holder to reproduce the material. To view a copy of this license, visit http:// creativecommons.org/licenses/by/4.0/ 